\title{
A síndrome de burnout e suas representações entre profissionais de saúde
}

\section{Burnout syndrome and its representations among health professionals}

\section{Síndrome de agotamiento laboral (burnout) y sus representaciones entre profesionales de la salud}

\author{
Luciana Bezerra de Souza Gianasi* \\ Universidade do Estado do Rio de Janeiro - UERJ, Rio de Janeiro, Brasil \\ Denize Cristina de Oliveira** \\ Universidade do Estado do Rio de Janeiro - UERJ, Rio de Janeiro, Brasil
}

\begin{abstract}
RESUMO
O artigo tem como objetivo comparar as representações sociais do esgotamento profissional e do burnout, entre profissionais de saúde de um hospital público universitário do Rio de Janeiro. O presente trabalho é parte dos resultados de uma tese de doutorado, podendo ser caracterizado como um estudo descritivo, de caráter transversal, a partir de uma amostra de conveniência totalizando 99 sujeitos. O instrumento de coleta foi um questionário contendo a técnica de evocações livres, cuja análise se deu por meio do uso do software EVOC 2005 e da construção do quadro de quatro casas. Os resultados apontam a existência de uma única representação social da "síndrome de burnout" e do "esgotamento profissional", estruturada a partir dos termos estresse e cansaço que fazem parte do núcleo central. Pode-se concluir que os profissionais de saúde possuem uma representação social alicerçada em duas dimensões, uma física e outra psicológica, destacando a importância das condições de trabalho para as profissões de saúde, especialmente no campo cirúrgico. Os resultados poderão contribuir para o desenvolvimento do campo teórico do burnout, sobretudo para sua compreensão conceitual.
\end{abstract}

Palavras-chave: representação social, síndrome de burnout, profissional de saúde.

\begin{abstract}
The article aims to compare the social representations of Exhaustion Professional and Burnout, among health professionals in a public university hospital in Rio de Janeiro. This work is part of the results of a doctoral thesis, which may be characterized as a descriptive, cross-sectional profile, departing from a convenience sample, in a total of 99 individuals. The data collection instrument was a questionnaire using free evocation of words technique, analyzed through the EVOC 2005 software and construction of the four-digit chart. The results indicate a single social representation of "burnout syndrome" and "exhaustion professional", structured from the
\end{abstract}


terms stress and fatigue that are part of the central nucleus. It can be concluded that health professionals have a rooted social representation in two dimensions, one physical and other psychological, emphasizing the importance of the working conditions for health professions, especially in the surgical ward. The results may contribute to the development of theoretical field of burnout, especially for its conceptual understanding.

Keywords: social representation, burnout syndrome, health professional.

\begin{abstract}
RESUMEN
El artículo tiene como objetivo comparar las representaciones sociales de agotamiento profesionale y el Burnout, entre los profesionales de la salud de un hospital público universitario en Rio de Janeiro. Es parte de una tesis doctoral, con un carácter descriptivo, de corte transversal, por un total de 99 sujetos. El instrumento fue un cuestionario que contiene la técnica de evocación libre, cuyo análisis fue a través del software EVOC 2005 y la construcción del cuadro de cuatro casas. Los resultados apuntan a una sola representación social, de la "agotamiento profesionale' y "síndrome de burnout", estructurada por los términos estrés y la fatiga que forman parte del núcleo central. Se puede concluir que los profesionales de la salud tienen una representación social fundamentada en dos dimensiones física y psicológica, destacando la importancia de las condiciones de trabajo para los profesionales de la salud, especialmente en el campo quirúrgico. Los resultados contribuyen al desarrollo del campo teórico del síndrome de agotamiento laboral, sobre todo para la comprensión conceptual.
\end{abstract}

Palabras-clave: representación social, síndrome de agotamiento laboral, profesionales de la salud.

\title{
1 Introdução
}

Apontada como um fenômeno em expansão na atualidade, a síndrome de burnout, também conhecida como esgotamento profissional no Brasil, tem recebido diferentes denominações (GilMonte, 2005). A síndrome de "quemarse por el trabajo" é uma delas, como é mais conhecida na Espanha. Sua discussão como um fenômeno psicológico foi realizada por Freudenberger (1974), nos Estados Unidos, a partir da observação do comportamento de voluntários que prestavam seus serviços em uma instituição de pessoas usuárias de drogas.

A definição do burnout utilizada pela maioria dos estudos voltados para os profissionais de saúde (Carlotto \& Câmara, 2008; Hyeda \& Handar, 2011; Trindade, Lautert, Beck, Amestoy, \& Pires, 2010; Lima et al., 2007; Rojas \& Grisales, 2011; Tironi et al., 2009), sustenta-se na perspectiva tridimensional (Maslach, 1993; Maslach \& Jackson, 1981, 1986; Maslach, Schaufeli, \& Leiter, 2001). A exaustão emocional caracteriza-se por um sentimento forte de tensão emocional. Já a despersonalização é o resultado do desenvolvimento de atitudes negativas de dureza e de distanciamento dos profissionais em relação às pessoas que se beneficiam dos seus serviços. Por fim, a diminuição da realização profissional está associada ao sentimento 
de incompetência no trabalho (Maslach, 1993; Maslach et al., 2001). Embora a definição de burnout, sustentada pelo modelo tridimensional, apareça em grande parte dos nos estudos com os nos profissionais de saúde, Gil-Monte (2005) considera não haver um consenso sobre o que é e como se processa a síndrome, denotando a possibilidade da existência de lacunas teóricas e conceituais sobre o assunto.

Estudos realizados no âmbito dos profissionais de saúde concordam em considerar a síndrome como sendo multifatorial. A forma como se desenvolve o processo de burnout é bastante diversificada (Benevides-Pereira, 2002) e os modelos explicativos mais relevantes estão descritos em Gil-Monte (2005), o qual propõe um modelo alternativo para explicar o processo, denominado "modelo estrutural sobre antecedentes e consequências do burnout" (Gil-Monte, Peiró, \& Valcarcel, 1995).

Confirmando a premissa de que há dificuldade em se estabelecer um consenso entre os autores quanto à definição e modelos explicativos do burnout, Carlotto (2001) aponta a subdivisão das concepções teóricas em quatro grandes grupos, sendo elas: a clínica, a sóciopsicológica, a organizacional e a sócio-histórica ou psicossocial, destacando esta última como sendo a mais adotada. Quanto ao aspecto conceitual do burnout, verificou-se haver diferentes modelos e concepções teóricas, o que acaba sugerindo a necessidade de novos estudos, visando uma melhor compreensão sobre o tema.

Quanto à exposição dos profissionais de saúde ao burnout, a revisão de literatura reforça essa categoria como estando entre as mais afetadas, tanto no cenário internacional, quando na realidade brasileira. Esses estudos sugerem, ainda, aspectos negativos do burnout tanto para o profissional trabalhador, como para a organização do trabalho, e mais amplamente para os usuários dos serviços prestados por estes profissionais. Além disso, considerando o caráter epidemiológico da síndrome, também é possível supor que um maior número de pessoas possa ser acometido pelo burnout. (Albadejo et al., 2004; Benevides-Pereira, 2002; Dias, Queiroz, \& Carlotto, 2010; Gil-Monte \& Marucco, 2008; Gonçalves et al., 2011; Hyeda \& Handar, 2011; Lautert, 1997; Lorenz, Benatti, \& Sabino, 2010; Ruiz \& Rios, 2003; Santos \& Cardoso, 2010; Seligmann-Silva, 2011; Tamayo, Argolo, \& Borges, 2005)

Alguns resultados de pesquisas (Carvalho \& Magalhães, 2011; Jodas \& Haddad, 2009) mostraram que mesmo aqueles que não se encontram acometidos pelo burnout, apresentam predisposição para desenvolver esse problema. De forma geral, os resultados convergem para a incidência e para prevalência da síndrome nesses profissionais, como para o estado de vulnerabilidade dessa categoria ao estresse (Oliveira \& Chaves-Maia, 2008; Santos \& Cardoso, 2010), constituindo-se em um grupo vulnerável ao aparecimento do burnout. 
Gomes e Cruz (2004), fazendo referência à realidade portuguesa, colocam que há uma tendência pelo crescente aumento de pesquisas sobre o estresse e a saúde ocupacional nos mais variados contextos profissionais. Os autores supracitados complementam, afirmando que "no entanto, esta tendência não tem sido totalmente acompanhada pela Psicologia e pelos seus profissionais que parecem ignorar a relevância desta área para o interesse público" (Gomes \& Cruz, 2004, p.194). A referência feita pelos autores em relação aos psicólogos parece denotar o desconhecimento destes profissionais de saúde em relação à relevância do tema.

No Brasil, Lima et al. (2007) afirmam que

"a síndrome de burnout ainda é desconhecida para grande parte dos profissionais de saúde. É necessária maior divulgação, pois, se estes desconhecem as manifestações e as causas, não podem buscar formas efetivas de tratamento, bem como a prevenção e a intervenção" (p.140).

Diante do crescente aumento de pesquisas sobre o estresse $e$ a saúde ocupacional, nos mais variados contextos profissionais, é possível inferir o destacado desconhecimento por parte dos profissionais de saúde em relação ao burnout, podendo estar relacionado ao número insuficiente de pesquisas, tanto no nível internacional, quanto no nacional. Na Argentina, e no cenário internacional, Gil-Monte e Marucco (2008) colocam a escassez de estudos em burnout com pediatras, chamando a atenção para a necessidade de novos estudos. Sobre a necessidade de mais estudos sobre o tema, na realidade brasileira, Carlotto e Câmara (2008) consideram que "embora pesquisas sobre burnout tenham uma longa tradição na América do Norte e Europa, no Brasil ainda encontramos poucos estudos sobre essa temática" (p.154).

Carvalho e Magalhães (2011) acrescentam "no Brasil, a literatura encontrada nos bancos de dados utilizados não é vasta em relação ao burnout e sua prevalência" (p.208). Ribeiro, Gomes e Silva (2009) completam dizendo que:

a experiência de estresse e burnout tem sido apontada como uma das áreas de impacto negativo no bem estar físico e mental dos profissionais de saúde. Apesar disso, ainda faltam estudos que indiquem melhor o impacto dessas variáveis e que esclareçam quais os fatores pessoais e profissionais explicativos das diferenças no processo de gestão das adversidades e dos desafios laborais (p.1503).

No Brasil, Carlotto e Câmara (2008) demonstraram a necessidade de mais pesquisas referentes ao tema do burnout, em um estudo 
direcionado à análise da produção científica brasileira sobre a síndrome de burnout. Os autores supracitados verificaram ser incipiente a produção científica brasileira sobre a síndrome de burnout, tanto em quantidade, como nos resultados já obtidos, propondo mais estudos que pudessem investigar, principalmente, as relações entre burnout e os fatores associados. Benevides-Pereira (2010) na mesma linha de pensamento enfatiza que:

necessitamos de estudos e de pesquisas nacionais, se possível mais abrangentes, para conhecermos melhor nossa realidade e a forma como o burnout se insere em nossa cultura, além do desenvolvimento de estratégias eficientes tanto para a prevenção, como para o diagnóstico e o tratamento, visando ao auxílio dos que já se encontram em risco (p.25).

A afirmativa da autora pela necessidade de novos estudos sobre o tema, contribuindo para a prevenção, diagnóstico e tratamento do burnout, constitui-se um ponto vital para a compreensão do burnout na realidade dos profissionais de saúde.

A revisão de literatura para o levantamento dos estudos de burnout, com referencial teórico na Teoria da Representação Social (TRS), mostrou a escassez de estudos internacionais e nacionais envolvendo o burnout à luz da TRS. A síndrome de burnout não apareceu como objeto de pesquisa de representação social e sim como resultados de investigações cujos objetivos eram a verificação da RS de outros objetos, como "a psicologia do trabalho" (Santos, 2010). Somente um único estudo português (Marques-Pinto, 2001) apontou o burnout como objeto de RS, referindo-se ao burnout profissional em professores portugueses.

A teoria da representação social, pelos progressos alcançados no campo metodológico, tornou-se um campo de investigação avançado tecnicamente (Lahlou, 2011). Moscovici (2012) aponta que as representações sociais "são entidades quase tangíveis; circulam, se cruzam e se cristalizam continuamente através da fala, do gesto, do encontro no universo cotidiano" (p. 39). Moscovici (2003) acrescenta, sobre as representações sociais, que elas "se apresentam como uma rede de ideias, metáforas e imagens, mais ou menos interligadas livremente" (p. 210). Também podem ser compreendidas como "conjuntos dinâmicos, seu estatuto sendo o da produção de comportamentos e de relações com o ambiente, da ação que modifica uns e outros, e não a reprodução de comportamentos ou relações, como reação a um dado estímulo externo" (Moscovici, 2012, p.39). Lançando luz ao conceito de representação social, Jodelet (2001) propõe uma conceituação geral, apontando uma convergência quanto à natureza dos fenômenos representacionais, a partir da seguinte definição sintética: "uma forma de conhecimento, socialmente 
elaborada e partilhada, com um objetivo prático, e que contribui para a construção de uma realidade comum a um conjunto social" (p. 22). Enfatizando a dimensão cognitivo-estrutural das representações (Sá, 1998), a abordagem estrutural da TRS se baseia na teoria do núcleo central, analisando os processos em funcionamento nas representações sociais (Abric, 2005). A teoria do núcleo central aponta a representação social organizada por meio de dois subsistemas: um núcleo central e um sistema periférico, sendo que "se o núcleo central constitui, de algum modo, a cabeça ou o cérebro da representação, o sistema periférico constitui o corpo e a carne" (Abric, 2003, p.38).

Aprofundando, o núcleo central determina o significado, a organização interna e a estabilidade da representação social e é constituído pelos valores associados ao objeto representado (Abric, 2003). Já o sistema periférico, está mais associado às características individuais e seu papel "pode ser resumido em cinco funções: concretização, regulação, prescrição de comportamentos, proteção do núcleo central e personalização" (Abric, 2003, p.38). Quando comparado ao núcleo central, o sistema periférico "é bem menos limitante, ele é mais leve e flexível. É a parte mais acessível e mais viva da representação" (Abric, 2003, p.38).

Este estudo objetiva conhecer e comparar as representações sociais dos profissionais de saúde acerca da síndrome de burnout e do esgotamento profissional, entre profissionais de saúde de um hospital público do Rio de Janeiro.

\section{Método}

Para a análise do burnout, foi utilizada a base téorica e metodológica da Teoria da Representação Social em sua vertente estrutural, por meio da análise do núcleo central e do sistema periférico.

\subsection{Sujeitos, amostra e cenário}

Os sujeitos da pesquisa são 99 profissionais de saúde que atuam nas equipes da clínica cirúrgica de um hospital público, de atendimento geral e de natureza docente-assistencial, localizado na cidade do Rio de Janeiro. A escolha dos sujeitos foi realizada a partir de uma amostra de conveniência. A clínica cirúrgica tem sido apontada como uma das áreas da saúde que apresenta frequência significativa de casos de manifestação de estresse e burnout (Lima et al. 2007; Rodrigues, 2008; Schmidt, Dantas, Marziale, \& Laus, 2009). No mesmo pensamento, a prática em anestesiologia, área médica que integra a clínica cirúrgica, tem sido apontada como uma atividade estressante e desgastante (Calumbi, Amorim, Maciel, Filho, \& Teles, 
2010; Neto, 2006; Torres et al., 2006).

Adotaram-se como critérios de inclusão dos participantes: profissionais de saúde de nível superior; integrantes da equipe cirúrgica; vinculados às enfermarias cirúrgicas do hospital. Além disso, participaram da pesquisa os profissionais em pleno desempenho das suas atividades, sendo excluídos aqueles já aposentados, ou que estivessem afastados, ou ainda com licença médica. Tomando-se estes critérios, integraram a amostra médicos cirurgiões das diversas especialidades, médicos anestesistas e enfermeiros.

\subsection{Coleta dos dados}

A coleta de dados foi realizada no período compreendido entre fevereiro e maio de 2014, utilizando-se o instrumento e procedimento a seguir descritos.

Para a coleta de dados os profissionais foram abordados individualmente, no centro cirúrgico ou nas respectivas secretarias e enfermarias das unidades cirúrgicas do hospital. Estes profissionais foram convidados a participar da pesquisa, assinando o termo de consentimento livre e esclarecido.

O questionário da evocação livre compôs um protocolo de pesquisa formado por outras duas partes, sendo elas um questionário de identificação sócio, demográfica e profissional e uma escala de caracterização do burnout. O questionário de evocação livre e as demais partes do protocolo de pesquisa foram respondidas individualmente, de forma autoaplicada, durante o expediente de trabalho.

A técnica da evocação livre é utilizada no levantamento dos conteúdos e da estrutura da representação social (Oliveira, Marques, Gomes, Teixeira, \& Amaral, 2005). Com essa técnica pretendese conhecer os conteúdos e a estrutura da representação social da síndrome de burnout, no âmbito dos profissionais de saúde. A técnica da evocação livre está em conformidade com o que preconiza a perspectiva teórica estrutural da representação social (Abric, 1998; $2001 ; 2003 ; 2005)$.

Segundo Oliveira et al. (2005), no domínio das representações sociais, a técnica da evocação livre consiste em:

pedir aos indivíduos que produzam todas as palavras ou expressões que possam imaginar a partir de um ou mais termos indutores, ou ainda em solicitar um número específico de palavras, seguindo-se de um trabalho de hierarquização dos termos produzidos, do mais para o menos importante (p.575). 
Em conformidade com a proposição supracitada realizou-se a coleta das evocações na qual o sujeito procedeu à leitura dos termos indutores, registrando no instrumento um número específico de 05 (cinco) palavras, para cada termo indutor. Os termos indutores empregados foram, nessa ordem, "síndrome de burnout" e "esgotamento profissional".

Quanto aos aspectos éticos, o projeto foi aprovado por meio do parecer consubstanciado de número 500. 118, do Comitê de ética em pesquisa (CEP), em 13 de novembro do ano de 2013.

\subsection{Tratamento e análise dos dados da evocação livre}

O tratamento dos dados da evocação se deu por meio do recurso informático Ensemble de Programmes Permettant L'analyse des Evocations - EVOC (Vergès, 1999), versão 2005. Os passos do tratamento dos dados e do funcionamento do software estão descritos em Oliveira (2000) e Oliveira et al. (2005). O tratamento dos dados consiste, resumidamente, em: digitar todas as evocações em sua forma bruta; realizar as correções ortográficas e padronizar as palavras e os termos evocados; transformar os arquivos trabalhados em formato adequado para serem processados pelo EVOC que calculará e informará as frequências simples, a frequência média das palavras, as frequências médias ponderadas das palavras e a média das ordens médias ponderadas do conjunto dos termos evocados. Tais etapas acontecem seguindo-se alguns programas agregados ao EVOC, sendo os primeiros denominados Lexique e Trievoc e o último denominado Catini (Oliveira et al., 2005).

A técnica do "quadro de quatro casas" (Vergès, 1994; Sá, 1998) orientou as análises das evocações. Tomando-se como referência Oliveira et al. (2005) a construção desse quadro de distribuição permite apontar hipóteses de centralidade, através dos elementos do núcleo central e dos elementos periféricos de uma representação social. Os critérios adotados para a distribuição dos termos nos quadrantes são a frequência média de ocorrência das palavras e a média das ordens médias de evocação das palavras, formandose valores que no seu cruzamento determinam os limites dos quadrantes.

Após a construção do quadrante, procederam-se à denominação e interpretação dos mesmos, tendo-se a seguinte configuração: caracterizando o núcleo central encontram-se as palavras situadas no quadrante superior esquerdo; formam a $1^{\text {a }}$ periferia as palavras situadas no quadrante superior direito, caracterizando os elementos periféricos mais importantes; constituindo os elementos de contraste, estão as palavras que se encontram no quadrante inferior esquerdo, sendo considerados elementos importantes pelos sujeitos, mesmo tendo uma baixa frequência; e por fim, como $2^{\mathrm{a}}$ periferia, àquelas 
palavras encontradas no quadrante inferior direito, sendo os elementos menos frequentes e menos importantes (Oliveira et al., 2005).

\section{Resultados e discussão}

\subsection{A representação social da "síndrome de Burnout"}

O produto das evocações ao primeiro termo indutor foi distribuído em quatro casas, como mostra a figura 1 , seguindo-se os critérios de frequência média e ordem média das evocações produzidas. A frequência mínima definida como ponto de corte, a partir do qual os termos passam a compor os quadrantes, foi 6 (seis). A partir desta definição, calculou-se a frequência média que foi 15 (quinze) e a ordem média das evocações (OME) de 2,9, para o total de 99 (noventa e nove) profissionais de saúde.

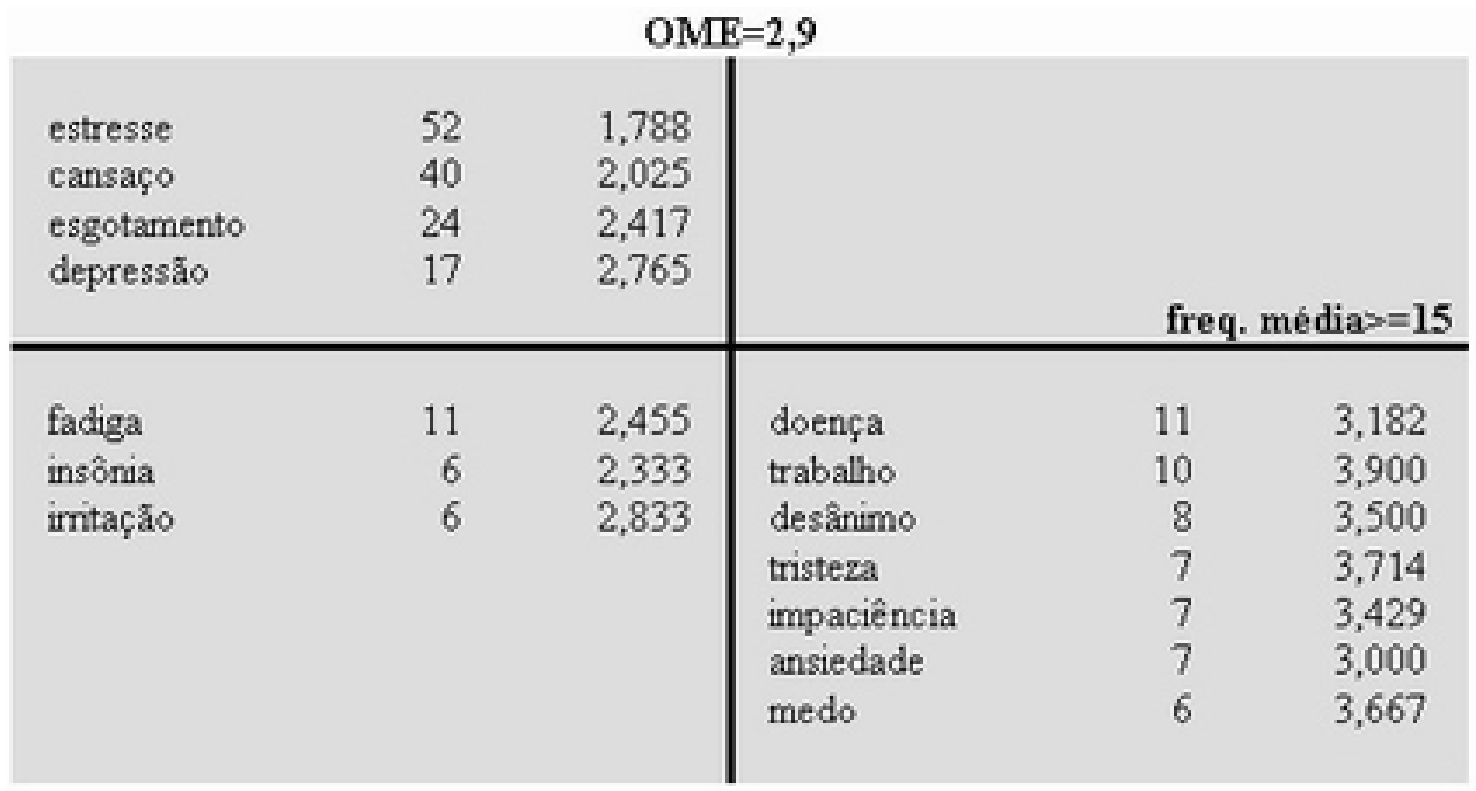

Figura 1. Quadro de quatro casas ao termo indutor "burnout". Rio de Janeiro, 2013.

Observando-se a estruturação das representações sociais do "burnout", por meio do quadro de quatro casas, pode-se observar que a hipótese de centralidade recai sobre as seguintes cognições, por ordem decrescente de frequência: cansaço, depressão, esgotamento e estresse. Destaca-se que esses termos são apontados como hipótese de centralidade por não ter sido realizado teste de centralidade dos mesmos, o que se apresenta como prática de pesquisa comum nos trabalhos publicados no Brasil, e também no exterior, nos últimos anos orientados pela abordagem estrutural das 
representações.

As palavras formadoras do núcleo central ocupam o quadrante superior esquerdo do quadro e recebem destaque pelo maior número de vezes que apareceram no corpus. Cabe salientar que o significado do "burnout" é fortemente marcado por elementos negativos, dentre os quais, o cansaço e o estresse foram os termos com maior frequência e os mais prontamente evocados. Na palavra cansaço, que tem a maior frequência e a que foi mais prontamente evocada, evidencia-se uma dimensão imaginética, expressando o componente físico da síndrome.

É provável que o aparecimento do cansaço reflita as características do trabalho em saúde, marcadas por muitas horas dedicadas ao trabalho, pelo acúmulo de vínculos empregatícios e pela carga horária extensa. Também se podem citar como exemplos, as longas horas de trabalho em pé da equipe cirúrgica, na realização de cirurgias longas, como também, o grande esforço físico despendido no manejo com o paciente.

Já a palavra estresse, revela a dimensão conceitual do fenômeno, já que a síndrome pode ser entendida como sendo uma cronificação do estresse. O aparecimento desta palavra denota o conhecimento/ esclarecimento que esses profissionais possuem acerca do burnout, confrontando uma hipótese inicial do trabalho, apoiada no referencial teórico, acerca do desconhecimento dos profissionais em relação ao burnout, mesmo sendo uma categoria marcada fortemente pela incidência da síndrome.

No que se refere à análise dos elementos da primeira periferia, localizados no quadrante superior direito, é possível observar a ausência de palavras neste quadrante. É provável que o esvaziamento deste quadrante tenha sido provocado pela convergência maior de palavras nos quadrantes da zona de contraste e segunda periferia, localizados nos quadrantes inferiores, esquerdo e direito, respectivamente, característica essa comum a dicionários que polarizam os léxicos em extremos, muito ou pouco citados.

A zona de contraste é formada pelas palavras fadiga, insônia e irritação, sendo fadiga a palavra com maior frequência e a mais prontamente evocada. Da mesma forma que na palavra cansaço em destaque no núcleo central, fadiga reflete uma dimensão física do burnout, podendo constituir-se como reflexo das características do trabalho.

Os elementos da segunda periferia são ansiedade, desânimo, doença, impaciência, medo, trabalho e tristeza. De maneira geral, estas palavras refletem uma dimensão objetiva e uma segunda afetivoatitudinal relacionada ao trabalho e à doença. Salienta-se a dimensão afetiva predominante nas palavras desânimo, medo e tristeza. Considera-se que a realidade do trabalho no hospital e fora dele e as situações negativas de trabalho, como por exemplo, o medo de 
errar e a perenidade da vida na sala de cirurgia podem contribuir para o sentimento de medo. Além disso, situações que extrapolam a realidade de trabalho do hospital e tornam-se características do diaa-dia nas grandes cidades, decorrentes da violência urbana, como o medo no deslocamento de casa para o trabalho, também podem levar ao sentimento de medo.

Nas palavras evocadas foi possível perceber as dimensões conceituais associadas ao fenômeno, demonstrando que os profissionais de saúde possuem uma representação social estruturada acerca da síndrome de burnout, que incorpora o esgotamento profissional, conforme será demonstrado a seguir. Além disso, os elementos evocados evidenciaram uma dimensão imagética da representação social do fenômeno, expressando o componente físico da síndrome, refletindo as características do trabalho em saúde. Finalmente os léxicos expressam dimensões afetivo/atitudinais da representação incorporados à síndrome de burnout.

\subsection{A representação social do "esgotamento profissional"}

Reportando-se à análise do termo indutor "esgotamento profissional" (Figura 2) definiu-se como pontos de corte para a construção do quadrante: frequência mínima 5 (cinco); frequência média 11 (onze); ordem média das evocações (OME) 2,9.

É possível perceber um núcleo central muito semelhante ao observado no termo indutor "síndrome de burnout", permitindo levantar a hipótese de que se trata de uma mesma representação. Para Abric (1998, p.38) "duas representações serão consideradas idênticas se forem organizadas em torno de um mesmo núcleo central".

\begin{tabular}{|c|c|c|c|c|c|}
\hline \multicolumn{6}{|c|}{$\mathrm{OME}=2,9$} \\
\hline cansaço & 37 & 1,649 & impaciência & 13 & 3,769 \\
\hline estresse & 35 & 2,200 & depressão & 11 & 3,000 \\
\hline desânimo & 13 & 2,692 & & & \\
\hline & & & & \multicolumn{2}{|c|}{ freq, média $>=11$} \\
\hline doenca & 6 & 2833 & irritabilidade & 9 & 3,333 \\
\hline insônia & 6 & 2,600 & férias & 6 & 3,500 \\
\hline fadiga & 6 & 2,500 & ansiedade & 6 & 3,333 \\
\hline excesso-traballho & 6 & 2,600 & desatençăo & 5 & 3,600 \\
\hline
\end{tabular}

Figura 2. Quadro de quatro casas ao termo indutor "esgotamento profissional". Rio de Janeiro, 2013.

Os léxicos revelam um conteúdo visivelmente negativo no suposto núcleo central do "esgotamento profissional", sendo o cansaço e o 
estresse os termos com maior frequência e os mais prontamente evocados. Apesar da aproximação dos dois termos pela semelhança entre seus núcleos centrais, a representação social do "esgotamento" evidencia diferenças.

No "esgotamento profissional" parece haver uma maior referência ao trabalho e suas consequências, com os termos doença e excessotrabalho na zona de contraste e férias no sistema periférico. Essa aproximação concreta ao trabalho pode estar relacionada à maior familiaridade que o termo na língua portuguesa possui, quando comparado ao termo em inglês burnout.

Infere-se que na análise comparativa dos dois termos indutores, considerando seus elementos centrais e periféricos, emergiram semelhanças no tocante ao conteúdo predominantemente negativo dos seus elementos constituintes, além da semelhança dos termos presentes no núcleo central, sendo os dois elementos com maior frequência e mais prontamente evocados o estresse e o cansaço. Um ponto de distinção entre as representações dos dois termos referese ao significado do sistema periférico de ambas, em que a dimensão relativa do trabalho, no seu sentido mais objetivo, é marcante na representação do esgotamento profissional.

\section{Conclusão}

A análise das representações sociais acerca do fenômeno burnout é importante para uma melhor compreensão da sua dimensão conceitual e fenomênica. Pode-se concluir que, diante dos resultados da pesquisa apresentados, os profissionais de saúde possuem uma representação estruturada acerca do fenômeno burnout associada às dimensões psicológica, afetiva e física.

Neste artigo, procurou-se comparar as representações sociais produzidas a partir de dois termos indutores referentes ao tema em tela, sendo eles "síndrome de burnout" e "esgotamento profissional". A partir dessa comparação, foram observadas semelhanças e diferenças nos conteúdos representacionais. Como principal semelhança, verificou-se que os profissionais de saúde possuem uma representação social estruturada acerca do burnout que se assemelha àquela do esgotamento profissional, podendo-se levantar a hipótese de se tratar de uma mesma representação. No entanto, essa constatação exigiria a aplicação de testes de centralidade, o que não foi realizado neste estudo.

Considera-se que o efeito de ordem no uso de dois termos indutores pode se colocar como uma limitação deste estudo, no entanto, o seu uso se justifica pela premissa adotada neste estudo de que o termo burnout poderia não ser familiar a todos os entrevistados e, também, pelo objetivo do estudo de comparar os conteúdos representacionais 
resultantes dos dois estímulos. Além disso, novos estudos devem ser realizados no sentido de confirmar a centralidade dos elementos da representação social, utilizando-se outras formas de análise complementares ao "quadro de quatro casas", como a técnica de "Similitude", descrita em Oliveira et al. (2005) ou as técnicas específicas de confirmação da centralidade, como os esquemas cognitivos de base, a indução por cenário ambíguo, conforme proposto por Abric (2001).

Os resultados encontrados contribuem para o desenvolvimento do campo teórico do burnout, sobretudo para sua compreensão conceitual, lançando luz para o entendimento de aspectos referentes ao trabalho dos profissionais de saúde. A possível repercussão em nível da prevenção e melhoria da saúde desses profissionais pode ecoar positivamente também nos serviços em saúde oferecidos à sociedade.

\section{Referências}

Abric, J. (1998). A abordagem estrutural das representações sociais. In A. S. P. Moreira, \& D. C. Oliveira (Orgs.). Estudos Interdisciplinares de Representação Social (pp. 27-38). Goiânia: $A B$.

Abric, J. (2001). Práticas sociales y representaciones. Paris: Press Universitaires de France.

Abric, J. (2003). Abordagem estrutural das representações sociais: desenvolvimentos recentes. In P. H. F. Campos, \& M. C. F. Loureiro (Orgs.). Representações Sociais e Práticas Educativas (pp. 36-47). Goiânia: UCG.

Abric, J. (2005). A zona muda das representações sociais. In D. C. Oliveira, \& P. H. F. Campos (Orgs.). Representações Sociais, uma Teoria sem Fronteiras (pp. 23-34). Rio de Janeiro: Editora Museu da República.

Albaladejo, R., Villanueva, R., Ortega, P., Astasio, P., Calley, M. E., \& Dominguez, M. E. (2004). Síndrome de Burnout en el personal de enfermería de un hospital de Madrid. Revista Española de Salud Pública, 78(4), 505-516.

Benevides-Pereira, A. M. T. (2002). Burnout: quando o trabalho ameaça o bem-estar do trabalhador. São Paulo: Casa do Psicólogo.

Benevides-Pereira, A. M. T (2010). Burnout: uma tão conhecida desconhecida síndrome. In C. T. Gisele, L. Machado, \& F. P. N. Sobrinho (Orgs.). A Síndrome de Burnout em Professores do Ensino Regular: Pesquisa, Reflexões e Enfrentamento (pp. 3945). Rio de Janeiro: Cognitiva. 
Calumbi, R. A, Amorim, J. A., Maciel, C. C., Filho, O. D., \& Teles, A. J. F. (2010). Avaliação da qualidade de vida dos anestesiologistas da cidade do Recife. Revista Brasileira de Anestesioogia, 60(1), 42-51.

Carlotto, M. S. (2001). Síndrome de burnout: um tipo de estresse ocupacional. Caderno Universitário, 18(1), 4-11.

Carlotto, M. S., \& Câmara, S. G. (2008). Análise da produção científica sobre a Síndrome de Burnout no Brasil. Psico, 39(2), 152-158.

Carvalho, C. G., \& Magalhães, S. R. (2011). Síndrome de burnout e suas consequências nos profissionais de enfermagem. Revista da Universidade Vale do Rio Verde, 9(1), 200-210.

Dias, S., Queirós, C., \& Carlotto, M. S. (2010). Síndrome de burnout e fatores associados em profissionais da área da saúde: um estudo comparativo entre Brasil e Portugal. Aletheia, 32(1), 421.

Freudenberger, H. J. (1974). Staff burn-out. Journal of Social Issues, 30(1), 159-165.

Gil-Monte, P. R. (2005). El síndrome de quemarse por el trabajo (burnout): Uma enfermidad laboral em la sociedad del bienestar. Madrid, España: Pirámide.

Gil-Monte, P. R., \& Marucco, M. (2008). Prevalencia del "síndrome de quemarse por el trabajo" (Burnout) en pediatras de hospitales generales. Revista de Saúde Pública, 42(3), 450-456.

Gil-Monte, P. R., Peiró, J. M., \& Valcárcel, P. (1995). A causal model of burnout process development: An alternative to Golembiewski and Leiter models. In VII European Congress of Work and Organizational Psychology. Resumos (p. 38). Györ: Hungría.

Gomes, A. R., \& Cruz, J. F. A. (2004). A experiência de stresse e "burnout" em psicólogos portugueses. Psicologia: Teoria, Invetigação e Prática, 1(2), 193-212.

Gonçalves, T. B., Leitão, A. K. R., Botelho, B. S., Marques, R. A. C, Hosoume, V. S. N., \& Neder, P. R. B. (2011). Prevalência de síndrome de burnout em professores médicos de uma universidade pública em Belém do Pará. Revista Brasileira de Medicina do Trabalho, 9(2), 85-89.

Hyeda, A., \& Handar, Z. (2011). Avaliação da produtividade na síndrome de burnout. Revista Brasileira de Medicina do Trabalho, 9(2), 78-84.

Jodas, D. A., \& Haddad, M. D. C. L. (2009). Síndrome de burnout em trabalhadores de enfermagem de um pronto socorro de hospital universitário. Acta Paulista de Enfermagem, 22(2), 192-7.

Jodelet, D. (2001). As representações sociais. Rio de Janeiro, RJ: EdUERJ. 
Lahlou, S. (2011). Difusão de representações e inteligência coletiva distribuída. In A. M. O. Almeida, M. F. S., Santos, \& Z. A. Trindade (Orgs.). Teoria das Representações Sociais, 50 anos (pp. 59-97). Brasília, DF: Technopolitik.

Lautert, L. (1997). O desgaste profissional: estudo empírico com enfermeiras que trabalham em hospitais. Revista Gaúcha de Enfermagem, 18(2), 133-44.

Lima, F. D., Buunk, A. P., Araújo, M. B. J., Chaves, J. G. M., Muniz, D. L. O., \& Queiroz, L. B. (2007). Síndrome de Burnout em residentes da Universidade Federal de Uberlândia-2004. Revista Brasileira de Educação Médica, 31(2), 137-46.

Lorenz, V. R., Benatti, M. C. C., \& Sabino, M. O. (2010). Burnout e estresse em enfermeiros de um hospital universitário de alta complexidade. Revista Latino Americana de Enfermagem, 18(6), 1-8.

Marques-Pinto, A. (2001). Burnout Profissional em Professores Portugueses: Representações Sociais, Incidência e Preditores. Tese de Doutorado não-publicada, Universidade de Lisboa, Lisboa, Portugal.

Maslach, C. (1993). Burnout: A multidimensional perspective. In W. B. Schaufeli, C. Maslach, \& T. Marek (Orgs.). Professional burnout: Recent developments in theory and research (pp. 134145). Washington, DC: Taylor \& Francis.

Maslach, C., \& Jackson, S. E. (1981). The measurement of experienced burnout. Journal of Organizational Behavior, 2(2), 99-113.

Maslach, C., \& Jackson, S. E. (1986). Maslach Burnout Inventory Manual. Palo Alto, CA: Consulting Psychologists Press.

Maslach, C., Schaufeli, W. B., \& Leiter, M. P. (2001). Job burnout. Annual Review of Psychology, 52(1), 397-422.

Moscovici, S. (2003). Representações Sociais: investigações em psicologia social. Petrópolis, RJ: Vozes.

Moscovici, S. (2012). A psicanálise, sua imagem e seu público. Petropolis, RJ: Vozes.

Neto, G. F. D. (2006). Stress e fadiga na segurança do ato anestésico: impacto no desempenho profissional. Rio de Janeiro: SAERJ.

Oliveira, D. C. (2000). Representações Sociais e Saúde Pública: a subjetividade como partícipe do cotidiano em saúde. Revista de Ciências Humanas, 4(2), 47-65.

Oliveira, D. C., Marques, S. C., Gomes, A. M. T., Teixeira, M. C. T. V., \& Amaral, M. A. D. (2005). Análise das evocações livres: uma técnica de análise estrutural das representações sociais. In A. S. Paredes (Org.). Perspectivas teórico-metodológicas em representações sociais (pp. 573-603). João Pessoa: Editora Universitária UFPB. 
Oliveira, L., \& Chaves-Maia, E. (2008). Saúde psíquica dos profissionais de saúde em hospitais públicos. Revista de Salud Pública, 10(3), 405-413.

Ribeiro, L., Gomes, A. R., \& Silva, M. C. M. (2009). Stresse ocupacional em profissionais de saúde: um estudo com médicos e enfermeiros portugueses. Estudos de Psicologia, 14(3), 239248.

Rodrigues, M. A. (2008). Stress e burnout na equipa multidisciplinar cirúrgica. Dissertação de mestrado não publicada, Universidade Aberta, Portugal.

Rojas B. M. L., \& Grisales, R. H. (2011). Burnout syndrome in professors from an academic unit of a Colombian university. Investigación y Educación en Enfermería, 29(3), 427-434.

Ruiz, C. O., \& Ríos, F. L. (2003). El burnout o síndrome de estar quemado en los profesionales sanitarios: revisión y perspectivas. International Journal of Clinical and Health Psicology, 4(1), 137-60.

Sá, C. P. (1998). A construção do objeto de pesquisa em representações sociais. Rio de Janeiro, RJ: EdUERJ.

Santos, E. C. M. (2010). Representações sociais da Psicologia do Trabalho: o olhar de formandos em Psicologia da Universidade Federal do Pará. Dissertação de mestrado, Universidade Federal do Pará, PA, Brasil.

Santos, A. F. O., \& Cardoso, C. L. (2010). Profissionais de saúde mental: estresse, enfrentamento e qualidade de vida. Psicologia: Teoria e Pesquisa, 26(3), 543-548.

Schmidt, D. R. C., Dantas, R. A. S, Marziale, M. H. P., \& Laus, A. M. (2009). Estresse ocupacional entre profissionais de enfermagem do bloco cirúrgico. Texto e Contexto em Enfermagem, 18(2), 330-337.

Seligmann-Silva, E. (2011). Trabalho e desgaste mental: o direito de ser dono de si mesmo. São Paulo, SP: Cortez.

Tamayo, M. R.; Argolo, J. C. T., \& Borges, L. O. (2005). Burnout em profissionais de saúde: um estudo com trabalhadores do município de Natal. In L. O. Borges (Org.). Os profissionais de saúde e seu trabalho (pp. 223-246). São Paulo: Casa do psicólogo.

Tamayo, M. R., \& Tróccoli, B. T. (2002). Exaustão emocional: relações com a percepção de suporte organizacional e com as estratégias de coping no trabalho. Estudos de Psicologia, 7(1), 37-46.

Tironi, M. O. S., Sobrinho, C. L. M, Barros, D. S., Reis, E. J. F., Filho, E. S. M., Almeida, A., \& Bitencout, A. (2009). Trabalho e síndrome da estafa profissional em médicos intensivistas de Salvador. Revista Associação Médica Brasileira, 55(5), 656-662. 
Torres, B. F., Pérez, L. M. R., Vélez, A. G., Rodríguez, T. R., Guillén, A. G., \& Béjar, M. (2006). Prevalencia del síndrome de Burnout en los anestesiólogos del Hospital Universitario Virgen Macarena de Sevilla. Revisista Espanhola de Anestesiologia, 53(6), 359362.

Trindade, L., Lautert, L., Beck, C. L. C., Amestoy, S. C., \& Pires, D. E. P. (2010). Estresse e síndrome de burnout entre trabalhadores da equipe de Saúde da Família. Acta Paulista de Enfermagem, 23(5), 684-9.

Vergès, P. (1994). Appoche du noyau central: proprietès quantitatives et structurales. In C. Guimelli (Org). Textes de base in sciences sociales: structures et transformation des representations sociales (pp. 233-253). Paris: Delachaux et Niestlé.

Vergès, P. (1999). Ensemble de programmes permettant l'analyse des évocations: Manuel version 2005. Aix-en-Provence: Laboratoire Méditerranéen de Sociologie.

\section{Endereço para correspondência Luciana Bezerra de Souza Gianasi}

Universidade do Estado do Rio de Janeiro

Programa de Pós Graduação em Psicologia Social - PPGPS

Rua São Francisco Xavier, $52410^{\circ}$ andar sala 10.009 bloco F, Maracanã, CEP 20550-013, Rio de Janeiro - RJ, Brasil

Endereço eletrônico: lucianabsg@yahoo.com.br

\section{Denize Cristina de Oliveira}

Universidade do Estado do Rio de Janeiro

Programa de Pós Graduação em Psicologia Social - PPGPS

Rua São Francisco Xavier, $52410^{\circ}$ andar sala 10.009 bloco F, Maracanã, CEP 20550-013, Rio de Janeiro - RJ, Brasil

Endereço eletrônico: dcouerj@gmail.com

Recebido em: 07/08/2014

Reformulado em: 23/09/2014

Aceito para publicação em: 24/09/2014

\section{Notas}

* Doutoranda do Programa de Pós-Graduação em Psicologia Social da Universidade do Estado do Rio de Janeiro, Rio de Janeiro, Brasil. Mestre em Psicologia Social.

** Professora do Programa de Pós-graduação em Psicologia Social da Universidade do Estado do Rio de Janeiro, Rio de Janeiro, Brasil. Doutora em Saúde Pública pela Universidade de São Paulo e Pós-Doutora em Psicologia Social. 